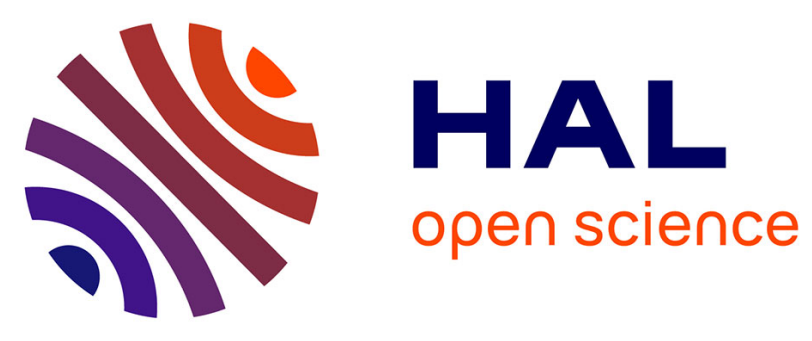

\title{
Cutaneous Mycobacterium chelonae infection in a patient with rheumatoid arthritis treated with glucocorticoids
}

Mervyn Mungroo, Etienne Ojardias, Adamah Amouzougan, Karima Boussoualim, Thierry Thomas, Hubert Marotte

\section{To cite this version:}

Mervyn Mungroo, Etienne Ojardias, Adamah Amouzougan, Karima Boussoualim, Thierry Thomas, et al.. Cutaneous Mycobacterium chelonae infection in a patient with rheumatoid arthritis treated with glucocorticoids. Joint Bone Spine, 2019, 86, pp.105 -. 10.1016/j.jbspin.2018.06.004 . hal-03484637

\section{HAL Id: hal-03484637 \\ https://hal.science/hal-03484637}

Submitted on 20 Dec 2021

HAL is a multi-disciplinary open access archive for the deposit and dissemination of scientific research documents, whether they are published or not. The documents may come from teaching and research institutions in France or abroad, or from public or private research centers.
L'archive ouverte pluridisciplinaire HAL, est destinée au dépôt et à la diffusion de documents scientifiques de niveau recherche, publiés ou non, émanant des établissements d'enseignement et de recherche français ou étrangers, des laboratoires publics ou privés.

\section{(ㄷ)(1) $\$$}

Distributed under a Creative Commons Attribution - NonCommerciall 4.0 International 


\section{Cutaneous Mycobacterium Chelonae infection in a patient with Rheumatoid Arthritis treated with glucocorticoids}

Mervyn Mungroo ${ }^{1}$, Etienne Ojardias ${ }^{1}$, Adamah Amouzougan ${ }^{1}$, Karima Boussoualim $^{1}$, Thierry Thomas ${ }^{1,2}$, and Hubert Marotte ${ }^{1,2,3}$

${ }^{1}$ Department of Rheumatology, North Hospital, University Hospital of St-Etienne, avenue Albert Raimond, 42270 Saint-Priest en Jarez, France

${ }^{2}$ SAINBIOSE, INSERM U1059, University of Lyon, Saint-Etienne, 42023 Saint Etienne, France

${ }^{3}$ CIC INSERM U1408, University of Lyon, Saint-Etienne, 42023 Saint Etienne, France

Corresponding author: Hubert Marotte

Department of Rheumatology, North Hospital, University Hospital of St-Etienne Avenue Albert Raimond 42270 Saint-Priest en Jarez, France

E-mail: hubert.marotte@chu-st-etienne.fr

Phone: +33-477-12-76-43

Fax: +33-477-12-75-77 
Clinical aspect of cutaneous mycobacterium chelonae infection in a 74 year-old female seronegative rheumatoid arthritis patient since 1988 treated with methylprednisolone $(25 \mathrm{mg}$ daily). Other relevant pathologies were type 2 diabetes, remitted non-Hodgkin lymphoma, and hypothyroidism. Physical examination revealed erythematous subcutaneous nodules, localized on her right arm, gradually spreading over a few months (Figure 1). No clinical or biological inflammation was reported. Cutaneous mycobacterium chelonae skin infection was confirmed by histology analysis and Lowenstein-Jensen culture. Skin lesions resolved after 9 months of clarithromycin and levofloxacin. Leflunomide was started 3 months after antibiotic beginning and rituximab at the skin lesion resolution. 
